\title{
Evaluación del impacto financiero en la gran empresa de
}

\section{Ambato}

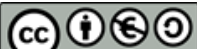

\section{Note of the financial impact on the large company of Ambato}

\author{
Marcelo Fernando Villalba Díaz. ${ }^{1}$
}

Recibido: 28-05-2020 /Aceptado: 21-06-2020 / Publicado: 03-07-2020

\begin{abstract}
.
DOI: https://doi.org/10.33262/concienciadigital.v3i3.1345

The objective of the research is to evaluate the financial impact on the Large Company, through the horizontal and vertical analysis of the years 2015 and 2016. This research is carried out based on the quantitative and qualitative paradigm, that is, based on data and facts of the objective reality to explain determine the results, and from the process- oriented qualitative paradigm, it is also supported from the descriptive, explanatory and correlational approaches, to relate the study variables from the perspective of the financial impact (financial indicators) measurable from the quantitative and qualitative. To establish financial guidelines that allow the optimization of the investment. With the results indicators were established. The assets of the company in relation to the two years analyzed there is a decrease of $\$ 624,066.00$, obtaining a proportional variation of $1.91 \%$. Sales revenue shows a decrease in 2016 compared to 2015 , with a percentage of $25.56 \%$, concluding that all companies have resorted to strategies to improve the development of their activities through the use of the proposed long-term strategic financial guidelines.
\end{abstract}

Keywords: Financial impact; financial indicators; strategic guidelines; quantitative analysis; qualitative analysis.

\section{Resumen.}

La investigación, tiene como objetivo evaluar el impacto financiero en la Gran Empresa, mediante el análisis horizontal y vertical de los años 2015 y 2016, La presente investigación se realiza fundamentado desde el paradigma cuantitativo y cualitativo, es decir basados en datos y hechos

\footnotetext{
${ }^{1}$ Escuela Superior Politécnica de Chimborazo, Riobamba, Ecuador, marcelo.villalbad@espoch.edu.ec
} 
de la realidad objetiva para explicar determinar los resultados, y desde el paradigma cualitativo orientado a los procesos, además se sustenta desde los enfoques descriptivo, explicativo y correlacional, para relacionar las variables de estudio desde la perspectiva del impacto financiero (indicadores financieros) medibles desde lo cuantitativo y cualitativo. Para establecer lineamientos financieros que permitan la optimización de la inversión. Con los resultados se establecieron indicadores. El Activo de la empresa en relación a los dos años analizados existe una disminución de \$624.066,00 obteniendo una variación proporcional del 1.91\%, El ingreso por ventas presenta una disminución en el 2016 con respecto al 2015, con un porcentaje del $25,56 \%$, concluyendo que todas las empresas han recurrido en estrategias para mejorar el desarrollo de sus actividades mediante el uso de los lineamentos estratégicos financieros a largo plazo propuestos.

Palabras claves: Impacto financiero; indicadores financieros; lineamientos estratégicos; análisis cuantitativo; análisis cualitativo.

\section{Introducción.}

Con la dinámica actual de mercado y los constantes cambios del ambiente competitivo, las medianas empresas desde esa prospectiva precisan cambiar, y en tiempos de cambios, es necesario un nuevo rumbo en la dirección empresarial; para ello, es importante planear estratégicamente para optimizar los recursos y el presupuesto, para la toma de decisiones, eficientes, además el uso y adaptación de las previsiones, mediante el análisis de alta gerencia de una manera válida de la información financiera con la finalidad de alcanzar tecnología e innovación como instrumentos importantes para la generación de rentabilidad.

La Corporación Financiera Nacional B.P asume un papel importante como soporte de las compañías. Sin embargo, después del otorgamiento del préstamo no se evidencia un estudio del impacto financiero ocasionado sobre las mencionadas empresas. Por otro lado la existencia de una escasa comunicación, coordinación, un deficiente acompañamiento de los directivos y una inadecuada orientación de los esfuerzos hacia la obtención del objetivo macro de las empresas, ejecutivos y parte operativa realizando funciones sin compromiso, ni motivación, es decir la mala comunicación con los gerentes de alto nivel, la inexistencia de planes de responsabilidad, carencia de indicadores financieros que permitan medir el desempeño económico y la no realización de medidas correctivas en las diferentes unidades, provoca que no se conozca con claridad el impacto financiero de los créditos otorgados por la Corporación Financiera Nacional B.P., a la gran empresa de la ciudad de Ambato durante el año 2016.

Con esta perspectiva las medianas y grandes empresas deben ejecutar el planeamiento financiero, basados en el conocimiento anticipado de todo el presupuesto e inversiones para desarrollo empresarial, permitiendo establecer, anticipadamente la nueva filosofía. Su práctica, permite aún, la identificación por parte de la empresa de necesidades como el aclaramiento o la posibilidad de expansión, la viabilidad de su presupuesto para el mercado actuante, la evaluación de posibilidad 
en la implementación de nuevos proyectos y sus costos, y la planificación de reservas para inversiones futuras.

Por consiguiente, el propósito de la investigación es evaluar el impacto financiero de la gran empresa que obtuvieron un préstamo en el CFN y como estas empresas logran llegar a ser competitivas. Dichas estrategias permiten expandir a la compañía hacia nuevos mercados fuera del territorio en el que se asientan. Además, con ello acrecienta la producción, edifica o consigue nuevas estructuras, realiza alguna transacción que la empresa vea como oportunidad o aprovecha una circunstancia que se presente.

En esta época de globalización, se evidencia la necesidad que poseen las empresas en su financiación, pues para cumplir todos sus cambios transcendentales como: la eficiencia, mejora de su rentabilidad, aumento de la productividad, entrar en nuevos mercados y convertirse en sociedades de clase mundial, requieren realizar fuertes inversiones originando de esta manera nuevas necesidades de financiamiento. Por tanto, el propósito es evaluar el impacto financiero en la gran empresa de la ciudad de Ambato para establecer lineamientos financieros que permitan la optimización de la inversión.

La evaluación de impacto financiero en los créditos otorgados a las grandes empresas es trascendental pues se analiza la situación de la compañía en todas las secciones de su actividad, dando énfasis a la localización de potencias o necesidades. De ahí la importancia de realizar un estudio que permita generar lineamientos estratégicos financieros de acción con respecto al contexto de inversión, convenientes con la realidad indagada. Al efectuar la evaluación del impacto financiero de los créditos otorgados por la CFN B.P. Se obtiene como resultado estrategias a largo plazo que promuevan el buen manejo de los recursos con respecto a la inversión solicitada por las empresas, por consiguiente, tiene como objetivo Evaluar el impacto financiero en la gran empresa de la ciudad de Ambato en el año 2016 en los créditos otorgados por la Corporación Financiera Nacional B.P.

Por todos los datos mencionados, la verificación del impacto financiero es un aporte sustancial para las grandes empresas; pues gracias a esta, se puede optimizar la inversión, el manejo adecuado de los recursos y sobre todo mantener la estabilidad y la rentabilidad.

\section{Metodología.}

La investigación busca dar soluciones a la situación problémica, para lo cual, plantea el problema desde el contexto del riesgo financiero y su impacto. Las empresas para llegar a ser competitivas requieren de créditos en busca de estrategias adecuadas de operación, inversión y financiamiento. Dichas maniobras permiten expandir a la compañía hacia nuevos mercados fuera del territorio en el que se asientan. Además, con ello acrecienta la producción, edifica o consigue nuevas estructuras, realiza alguna transacción que la empresa vea como oportunidad o aprovecha una circunstancia que se presente. Para ello se formula un problema: ¿Cómo la evaluación del impacto 
financiero en la gran empresa de la ciudad de Ambato en el año 2016 de los créditos otorgados por la Corporación Financiera Nacional B.P. permite establecer lineamientos financieros que permitan la optimización de la inversión?, bajo este parámetro se planteas los siguientes subproblemas de investigación:

Por consiguiente, con perspectivas de desarrollar la investigación se plantea el siguiente objetivo; Evaluar el impacto financiero en la gran empresa de la ciudad de Ambato en el año 2016 de los créditos otorgados por la Corporación Financiera Nacional B.P. para establecer lineamientos financieros que permitan la optimización de la inversión

La presente investigación se realiza fundamentado desde el paradigma cuantitativo y cualitativo, es decir basados en datos y hechos de la realidad objetiva para determinar los resultados, y desde el paradigma cualitativo orientado a los procesos, además se sustenta desde los enfoques descriptivo, explicativo y correlacional, para relacionar las variables de estudio desde la perspectiva del impacto financiero (indicadores financieros) medibles desde lo cuantitativo y cualitativo.

Para lo anterior, se diseñaron, calcularon y analizaron indicadores de liquidez, actividad y endeudamiento de las empresas del sector almacenamiento y actividades conexas en la gran empresa, con lo que se pudo evaluar la evolución, cambio y la significancia de la evaluación de los indicadores financieros a través de la técnica análisis discriminante en estas organizaciones

El grupo objetivo del estudio está conformado por las grandes empresas de la ciudad de Ambato que han obtenido créditos de la Corporación Financiera Nacional B.P. en el año 2015 y 2016. El número de establecimientos registrados se resumieron en tres establecimientos. Al ser un número menor a cien, se consideró el número total de población objeto de estudio, las cuales corresponden a las siguientes organizaciones: Bioalimentar Cia. Ltda., Incubadora Andina Incubandina Sociedad Anonima. Curtiduria Tungurahua S.A

El análisis fue desarrollado mediante la elaboración de un diagnóstico financiero sobre cada una de las grandes empresas mencionadas, resultados que posteriormente se utilizaron para la creación de lineamientos estratégicos a largo plazo para la optimización de la inversión de acuerdo a los créditos otorgados por la CFN B.P.

\section{Resultados.}

El diagnóstico financiero, radica en evaluar el impacto financiero que tiene el importe crediticio sobre el desarrollo de las grandes empresas de la ciudad de Ambato, dicha información, cuentas y balances, han sido recopiladas gracias a los datos otorgados por la Corporación Financiera Nacional B.P., en los años 2015 y 2016. El análisis cuantitativo y cualitativo radica en la información y concentración de activos y pasivos y patrimonio de las empresas objeto de estudio, 
en la cual se hace referencia con la aplicación de indicadores financieros. Por consiguiente, se interpreta en la figura 3.

Figura 1. Consolidación del análisis horizontal

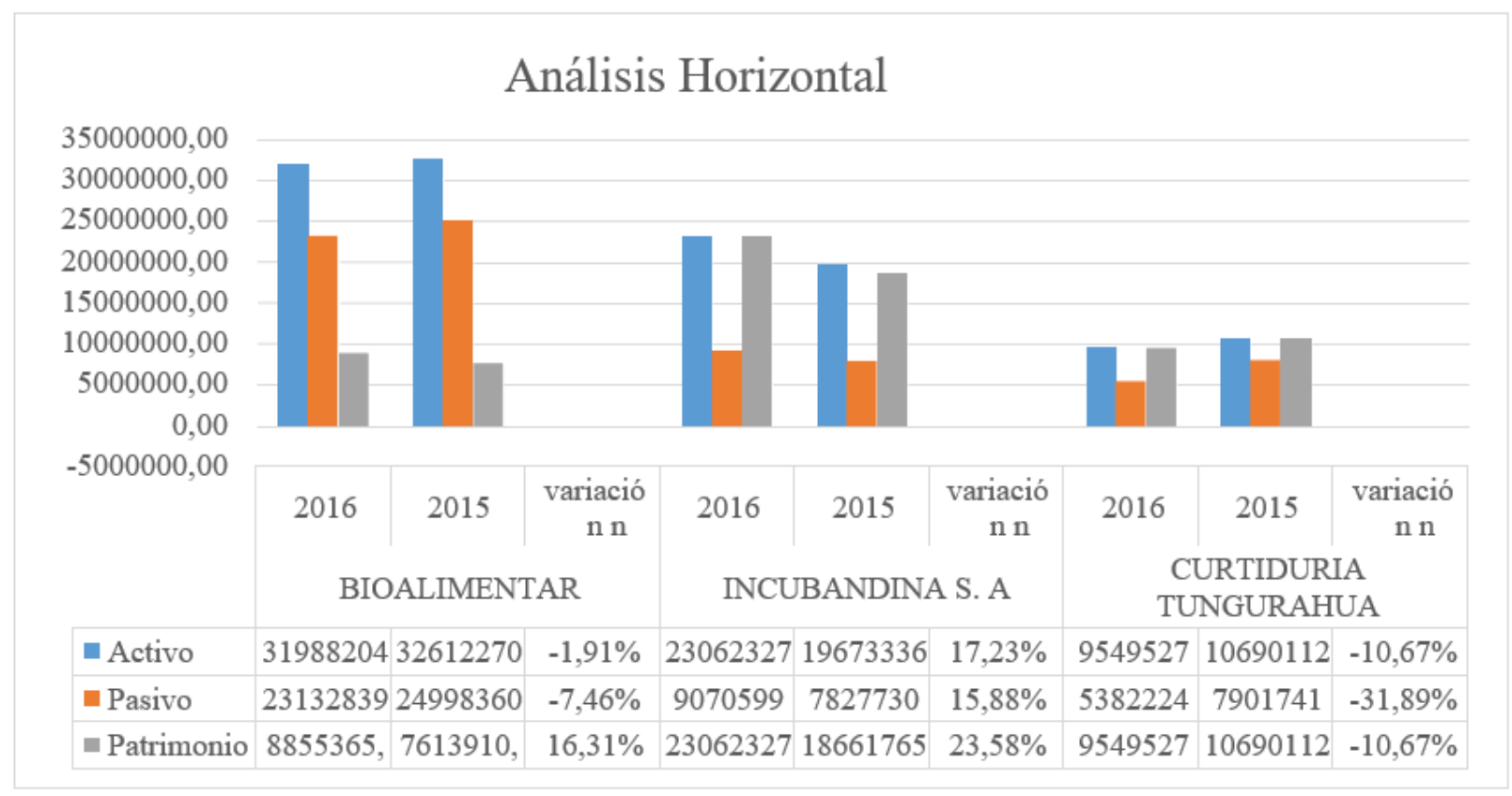

Fuente: Elaboración propia.

De acuerdo, Villalba (2019):

El Activo de la empresa en relación a los dos años analizados existe una disminución de $\$ 624.066,00$ obteniendo una variación proporcional del 1.91\%, donde la cuenta Seguros disminuye en $\$ 9.240 .532,00$ obteniendo un $99.15 \%$, reducción que pudo ser delimitada por la falta de desplazamientos de inversión bancaria en el año 2016. La cuenta de efectivo y equivalentes presenta una disminución en la variación del año 2016 con respecto al año 2015 de $\$ 284.868,00$ con un porcentaje del $14,31 \%$, efectivo que pudo ser utilizado para la creación de la nueva planta de producción ejecutada en el año 2016. La cuenta Inventarios en el año 2016 con relación al año 2015 tuvo uno variación de crecimiento en $\$ 7.883 .056,00$ que en forma porcentual representa el $768.92 \%$, necesidad que puede ser sustentada por requerir mayor inventario para la nueva planta de producción. Los impuestos por recuperar se elevaron de manera sustancial en el año 2016 con respecto al 2015 en \$ 1.170.156,00 dando un porcentaje del 994,78\%, representando a aquellas compras necesarias para la implementación de la nueva planta, de esta manera el crédito tributario de BIOALIMENTAR pudo elevarse (págs. 41-42).

Adicionalmente, Villalba (2019) establece que: 
Dentro del Activo no corriente entre los años 2016 y 2015 posee una disminución de \$92.919,00 con una variación proporcional de $0.62 \%$, reducción suscitada por las depreciaciones que presenta la propiedad planta y el equipo neto. Así también, se evidencia una baja en la cuenta activos tangibles de $\$ 11.318,00$ que representa una variación del 2,71\%, disminución que podría efectuarse porque la empresa adquirió franquicias, patentes, marcas o el know-how de sus activos intangibles. Entre el año 2016 y el año 2015, el Pasivo de la empresa BIOALIMENTAR presenta una disminución $\$ 1.865 .521,00$ que representa el 7.42\%, debido a que la cuenta Provisiones sufre una reducción de $\$ 461.340,00$ que en proporción representa el 100\% lo que podría denotarse que la empresa cancela en su totalidad el saldo registrado en el año 2015, pues este rubro presentaba una pérdida para la empresa. Dentro del Pasivo a Largo Plazo la cuenta Obligaciones con Instituciones Financieras tiene un incremento de \$3.752.738,00 obteniendo una variación proporcional de $242,95 \%$, este financiamiento es el que fue establecido con la CFN B.P. (págs. 42-44).

Figura 2. Consolidación del Análisis horizontal

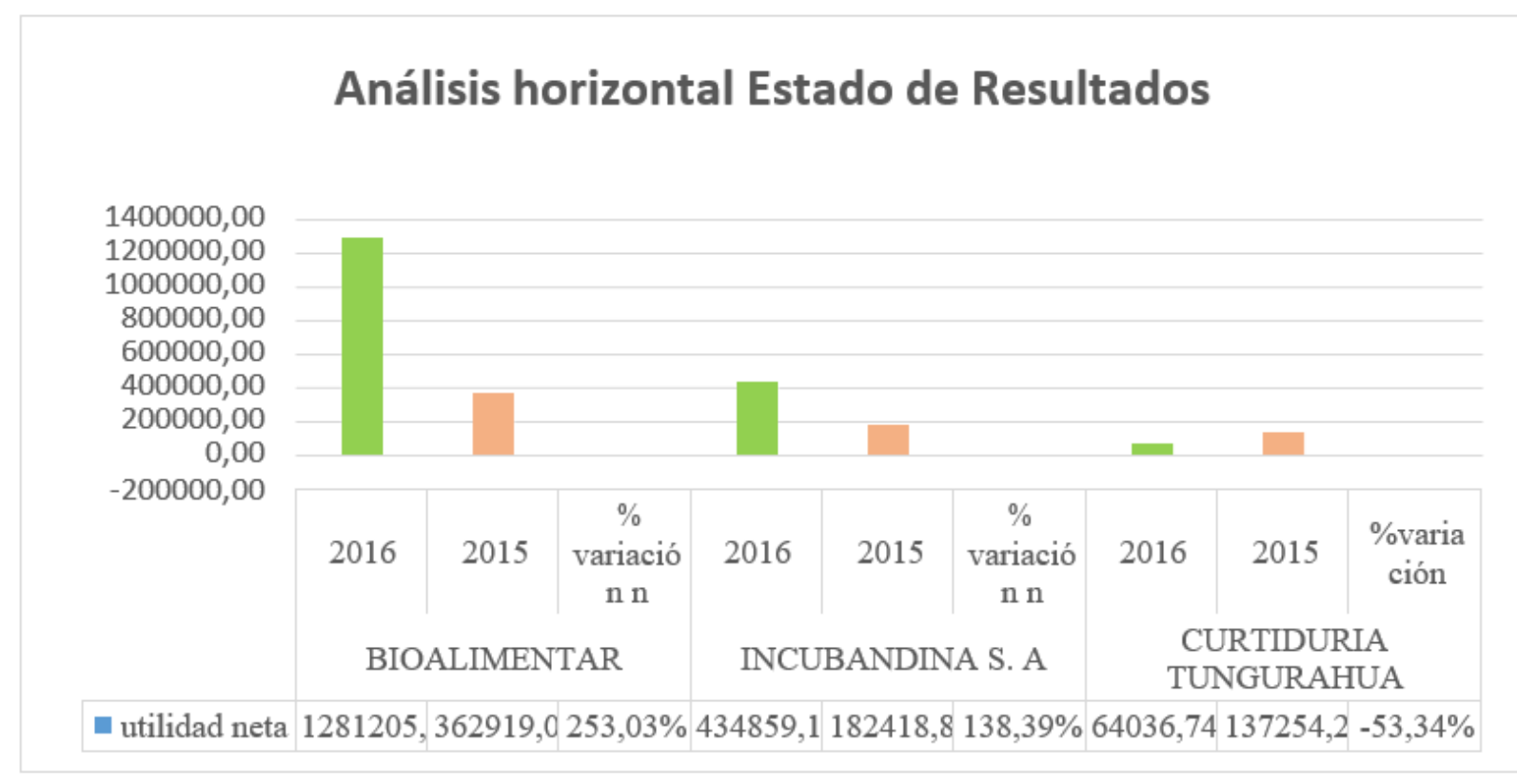

Fuente: Elaboración propia.

Según Villalba (2019):

El ingreso por ventas presenta una disminución en el 2016 con respecto al 2015, con un porcentaje del $25,56 \%$, dato que podría entenderse como un contante crecimiento de la competencia o el terremoto suscitado en el país. Los gastos de ventas tienen relación con el ingreso debido a la reducción significativa de un $29,11 \%$, situación que pudo suscitarse por la caída de ventas presentadas durante el año 2016. La utilidad operacional ha crecido en el año 2016 con respecto 
al 2015 con un porcentaje del 24,36\%, pudiendo evidenciarse por la reducción de gatos presentados en las ventas en un $29,11 \%$ y en los gastos de administración en un 22,67\%. La utilidad neta del año presenta un aumento de $\$ 814.471,00$ en el año 2016 con respecto al 2015, dando una variación proporcional de $224 \%$, pudiendo ser justificado porque la empresa presenta una disminución en sus gastos administrativos y costos de ventas demostrando un adecuado manejo de sus recursos (págs. 45-46).

Figura 3. Consolidación del Análisis vertical

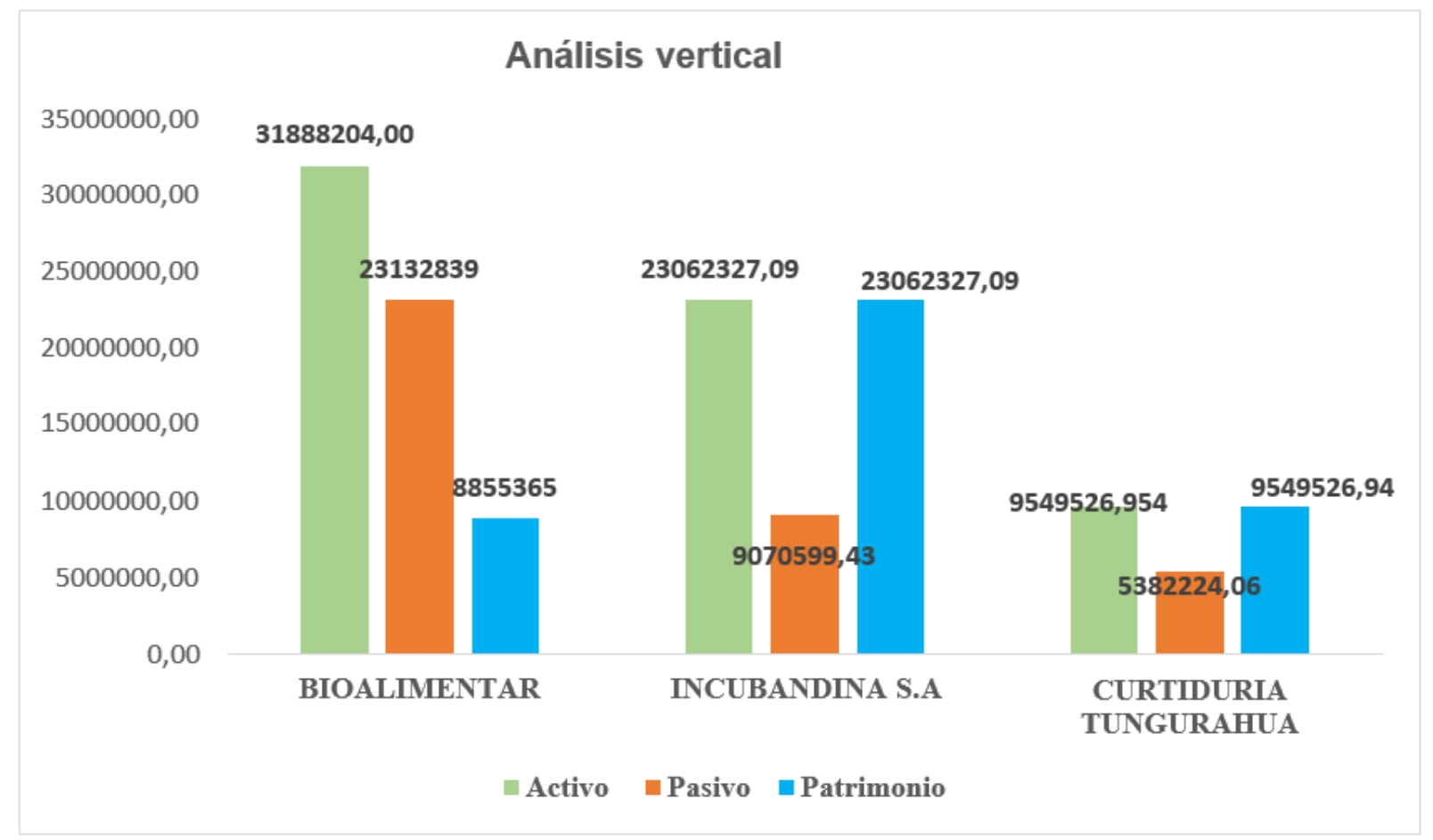

Fuente: Elaboración propia.

\section{Discusión.}

Para la realización de este estudio se utilizaron indicadores de liquidez, rentabilidad, y endeudamiento, de igual modo, a la hora de llevar a cabo la selección de indicadores financieros se partió de un conjunto amplio, intentando recoger los indicadores más significativos dentro del análisis de la situación financiera. Por otro lado, Sanchis, Gril y Heras (2003) y Cabarca (2003) señalan que estos indicadores analizados permiten enfocar los problemas de competitividad, excesiva exposición al riesgo, dificultades de crecimiento que de no corregirse pueden afectar la viabilidad de la empresa. Variables importantes, sobre todo cuando analizamos el efecto en la calidad en las empresas estudiadas. Debido a que, en el año 2016, las empresas deciden ampliar sus instalaciones para expandir su producto a nivel nacional e internacional. En el capital social no se registró ninguna variación entre los años estudiados, así como el aporte de las futuras 
capitalizaciones. Por otra parte, los resultados acumulados presentan incremento en el año 2016 con respecto al 2015.

En referencia a la capacidad de endeudamiento La razón deuda refleja la proporción de activos totales financiados por los acreedores de la empresa y mientras más alto sea su valor significa que los acreedores han aportado una gran suma de dinero para las operaciones empresariales esperando obtener beneficios.

La razón pasivo circulante y pasivo total, simplemente expresa la proporción de los pasivos totales correspondientes a las deudas o pasivos cuyo vencimiento es menor a un año; en tanto, la razón del pasivo a largo plazo y pasivo total, indica la proporción de los pasivos totales contraída por obligaciones a ser canceladas en un lapso de tiempo mayor a un año. En cuanto a la razón cobertura de intereses, arroja un valor que refleja la capacidad de la empresa para cancelar los intereses del endeudamiento incurrido, mientras más alto sea este valor mayor será la capacidad de la empresa para pagar.

En cuanto La rentabilidad constituye el resultado de las acciones gerenciales, decisiones financieras y las políticas implementadas en una organización. Fundamentalmente, la rentabilidad está reflejada en la proporción de utilidad o beneficio que aporta un activo, dada su utilización en el proceso productivo, durante un período de tiempo determinado; aunado a que es un valor porcentual que mide la eficiencia en las operaciones e inversiones que se realizan en las empresas.

A través del análisis financiero se pueden determinar los niveles de rentabilidad de las grandes empresas; pues, permite evaluar la eficiencia de la empresa en la utilización de los activos, el nivel de ventas y la conveniencia de efectuar inversiones, mediante la aplicación de indicadores financieros que muestran los efectos de gestionar en forma efectiva y eficiente los recursos disponibles, arrojando cifras del rendimiento de la actividad productiva y determinando si ésta es rentable o no. Entre estos indicadores se encuentran el rendimiento sobre las ventas, el rendimiento sobre los activos y el rendimiento sobre el capital aportado por los propietarios.

Lineamientos de selección para inversiones de largo plazo en las grandes empresas de Ambato.

La figura de notabilidad centralmente de la organización estratégica lo constituye la formulación de lineamientos, pues estos establecen normas generales que protegen la colocación de las operaciones con las estrategias señaladas, además de formarse como medidas de control sobre el desempeño y los resultados alcanzados por las grandes empresas de Ambato (Villalba, 2019, pág. 110).

\section{Utilización del indicador de liquidez.}

\section{Hallazgo.}


Una vez efectuado el análisis a los estados económicos, indicadores financieros históricos como los estados financieros proyectados de BIOALIMENTAR, empresa que tiene como actividad principal la nutrición animal como la humana y la producción agrícola, utilizando mecanismos como la investigación, el desarrollo, producción y la comunicación de soluciones integrales en todas las cadenas agroalimentarias, se observa una tendencia creciente, como se puede evidenciar en la tabla No 5-52 (Villalba, 2019, pág. 110).

Tabla 1. Indicadores liquidez históricos

\begin{tabular}{|l|r|r|r|r|}
\hline INDICES FINANCIEROS & \multicolumn{1}{|c|}{2013} & \multicolumn{1}{|c|}{2014} & \multicolumn{1}{|c|}{2015} & \multicolumn{1}{|c|}{$2016 * *$} \\
\hline LIQUIDEZ & & & & \\
\hline CAPITAL DE TRABAJO & $-\$ 3.889 .313$ & $-\$ 5.027 .455$ & $-\$ 5.026 .650$ & $\$ 238.469$ \\
\hline INDICE CORRIENTE & 0,78 & 0,81 & 0,79 & 1,01 \\
\hline INDICE ACIDO & 0,44 & 0,31 & 0,37 & 0,50 \\
\hline
\end{tabular}

Fuente: Elaboración propia.

Tabla 2. Indicadores liquidez proyectados

\begin{tabular}{|l|c|c|c|c|}
\hline INDICES FINANCIEROS & 2017 & $\mathbf{2 0 1 8}$ & $\mathbf{2 0 1 9}$ & $\mathbf{2 0 2 0}$ \\
\hline LIQUIDZZ & & & & $\$ 19.992 .586$ ( \\
\hline CAPITAL DE TRABAJO & $\$ 8.265 .478$ & $\$ 11.262 .827$ & $\$ 15.375 .396$ & 2,59 \\
\hline INDICE CORRIENTE & 1,75 & 1,92 & 2,28 & 1,87 \\
\hline INDICE ACIDO & 1,03 & 1,23 & 1,55 & \\
\hline
\end{tabular}

Fuente: Elaboración propia.

Analizando los niveles de liquidez registrado en la proyección por la empresa BIOALIMENTAR de la ciudad de Ambato es pertinente y oportuno formalizar una administración y programación financiera conveniente del circulante de manera que se proteja la viabilidad futura de la empresa (Villalba, 2019, pág. 111).

A continuación, se presentan algunos dinamismos que se consideraron para obtener los cambios en el indicador financiero de liquidez (Villalba, 2019, pág. 111):

- Contar con un presupuesto de Tesorería

Elaborar y conocer los flujos de fondos y la previsión de cobranza es importante para planificar cuando y como se realizarán la erogación y anticiparse a la eventual necesidad de fondos adicionales.

- Negociar los plazos de cobro y pago con clientes y proveedores

Establecer en la medida de lo posible una cadena de cobros y pagos que esté acompasada. A modo de ejemplo, si el plazo habitual de los cobros es a 60 días, establecer el mismo plazo para los proveedores (a excepción claro está, de las obligaciones con vencimiento legal establecido). 
- Tener una gestión eficiente de las cuentas a cobrar

Realizar una gestión eficiente de las cuentas a cobrar, permite concretar el pago en menores plazos, así como identificar en forma anticipada si hay riesgo de morosidad y tomar las medidas pertinentes para mitigarlo.

- Optimizar el stock

Tener un exceso de stock puede perjudicar seriamente la liquidez, así como también se puede producir roturas, deterioros y vencimientos que impidan la posterior venta de esos artículos (Villalba, 2019, pág. 112).

\section{Indicador de endeudamiento.}

\section{Hallazgo.}

En el análisis realizado a los estados financieros históricos y proyectados de la empresa BIOALIMENTAR, del sector de la producción de la ciudad de Ambato, durante los años 2015 a 2016 no se evidenciaron niveles razonables en este indicador; más por el contrario para los años proyectados este indicador demuestra una notable mejoría llegando a niveles razonables, esto se debe a las decisiones que los propietarios y/o administradores de la empresa efectuaron para el mejoramiento de la solvencia de la compañía (Villalba, 2019, pág. 112).

Tabla 3. Indicadores endeudamiento históricos

\begin{tabular}{|l|c|c|c|c|}
\hline INDICES FINANCIEROS & $\mathbf{2 0 1 3}$ & $\mathbf{2 0 1 4}$ & $\mathbf{2 0 1 5}$ & $\mathbf{2 0 1 6}$ ** \\
\hline SOLVENCIA & & & & \\
\hline ENDEUDAMIENTO & $77 \%$ & $80 \%$ & $77 \%$ & $73 \%$ \\
\hline
\end{tabular}

Fuente: Elaboración propia

Tabla 4. Indicadores endeudamiento proyectados

\begin{tabular}{|l|c|c|c|c|}
\hline INDICES FINANCIEROS & 2017 & 2018 & 2019 & 2020 \\
\hline SOLVENCIA & & & & \\
\hline ENDEUDAMIENTO & $54 \%$ & $51 \%$ & $46 \%$ & $41 \%$ \\
\hline
\end{tabular}

Fuente: Elaboración propia

\section{Aptitudes viables.}

Los pagos por gastos de intereses efectuados por la empresa BIOALIMENTAR son significativos es especial en los estados financieros históricos, más por el contrario para la simulación efectuada a los estados financieros y del análisis al indicador de endeudamiento obtenido, el mismo denota 
una considerable mejoría debido a la decisión de unificar las deudas que la empresa mantenía con la banca privada y la obtención de una línea de crédito a mayor plazo (Villalba, 2019, pág. 113).

\section{Lineamientos.}

- Analizar la estructura financiera, así como el nivel general de endeudamiento, de las distintas fuentes de financiación incluyendo autofinanciación y política de retención y/o reparto de utilidades.

- Ejecutar un estudio de opciones de endeudamiento considerando: el costo del financiamiento, escogiendo la posibilidad que beneficie a la organización tomando en cuenta la rentabilidad de la actividad de la empresa.

- Consolidación de deudas, que servirá a la empresa para, reducir sus gastos mensuales, administrar una sola deuda y que la misma sea más manejable para el futuro de la compañía (Villalba, 2019, pág. 113).

\section{Indicador de rentabilidad.}

El indicador de rentabilidad de la empresa BIOALIMENTAR del sector productivo de la ciudad de Ambato presenta un rendimiento que está ayudando apropiadamente al capital invertido por la organización; pues el índice manifiesta un porcentaje razonable. Se manifiesta también que la empresa en análisis ha presentado utilidades durante los años de estudio, aunque estas no sean considerables (Villalba, 2019, pág. 114).

Tabla 5. Indicadores rentabilidad históricos

\begin{tabular}{|c|c|c|c|c|}
\hline INDICES FINANCIEROS & 2013 & 2014 & 2015 & $2016 * *$ \\
\hline \multicolumn{5}{|l|}{ RENTABIUIDAD } \\
\hline RENTABILIDAD BRUTA & $129 \mathrm{~A}$ & 119 & 1394 & 1696 \\
\hline RENTABILIDAD OPERATIVA & 19 & 29 & 498 & 696 \\
\hline RENTABILIDAD NETA & 098 & 19 & 29 的 & 396 \\
\hline RENTABILIDAD SOBRE ACTIVOS & 098 & 19 & 39: & 496 \\
\hline $\begin{array}{l}\text { RENTABILIDAD SOBRE } \\
\text { PATRIMONIO }\end{array}$ & 094 & 59 & 149: & $15 \%$ \\
\hline
\end{tabular}

Fuente: Elaboración propia.

Tabla 6. Indicadores rentabilidad proyectados

\begin{tabular}{|c|c|c|c|c|}
\hline \multirow{2}{*}{\multicolumn{5}{|c|}{\begin{tabular}{|l}
$\mid$ INDICES FINANCIEROS \\
RENTABILIDAD
\end{tabular}}} \\
\hline & & & & \\
\hline RENTABILIDAD BRUTA & $18 \%$ & $19 \%$ & $20 \%$ & $21 \%$ \\
\hline RENTABILIDAD OPERATIVA & $9 \%$ & $10 \%$ & $12 \%$ & $13 \%$ \\
\hline RENTABILIDAD NETA & $4 \%$ & $5 \%$ & $6 \%$ & $7 \%$ \\
\hline $\begin{array}{l}\text { RENTABILIDAD SOBRE } \\
\text { ACTIVOS }\end{array}$ & $3 \%$ & $5 \%$ & $6 \%$ & $7 \%$ \\
\hline $\begin{array}{l}\text { RENTABILIDAD SOBRE } \\
\text { PATRIMONIO }\end{array}$ & $7 \%$ & $9 \%$ & $11 \%$ & $12 \%$ \\
\hline
\end{tabular}


Fuente: Elaboración propia.

\section{Aptitudes viables.}

En las empresas analizadas los indicadores de liquidez y la rentabilidad son índices contrastados, los activos corrientes tiene como propósito avalar desembolsos, pero no siempre esa inversión la hace en activos rentables. Es importante alcanzar una armonía entre uno y otro indicador (Villalba, 2019, pág. 114) .

\section{Lineamientos.}

- Incrementar márgenes de ganancia; en base a,

- Aumentar precios

- Reducir costos de los productos

- Llevar control de gastos, saber en qué se está gastando los recursos y dónde hacen falta.

- Incrementar la rentabilidad de la empresa.

- Mediante un plan estratégico de Marketing se fijará las estrategias de mercado de la empresa como son: métodos de distribución, calidad máxima en el producto, encontrar nichos de mercado, fijación de precios de acuerdo al valor en el mercado.

- Equilibrar las transacciones, mercados y canales de comercialización que son efectivamente convenientes y si están afines al propósito de rentabilidad de la empresa.

- Gestionar eficientemente el inventario de la empresa.

En definitiva, se deduce que el análisis financiero es una herramienta trascendental para determinar la situación financiera de una organización, de modo que se logre una gestión financiera eficiente; para ello resulta imprescindible llevar un control adecuado del uso de los activos y de los recursos financieros que están destinados para las inversiones, por lo cual se debe realizar un análisis minucioso de cada uso dado a los fondos disponibles, se trate de recursos propios o provenientes de terceros.

\section{Conclusiones:}

- Con el análisis del diagnóstico financiero, se puede medir la concentración del activo, pasivo y patrimonio sobre la estructura financiera de cada empresa, verificando aquellas cuentas que tienen mayor relevancia dentro de los estados financieros, es decir si la empresa posee una concentración mayor en su activo el desarrollo de sus actividades estará en un margen de normalidad. Por otra parte, cuando el pasivo es el que mayoritariamente prevalece, determina que se ha incurrido a un endeudamiento. Esta característica perimió determinar que las grandes empresas en estudio recurrieron a un financiamiento para el desarrollo adecuado de sus actividades. En tal efecto la evaluación de los indicadores financieros demuestran que el endeudamiento dentro de las grandes empresas es aceptable, puesto que la entidad financiera otorga préstamos para Capital de Trabajo, Activo fijo, y Apoyo Productivo Financiero, por ello los establecimientos al evaluar la alternativa de un endeudamiento de largo plazo, se están proyectando hacia un mejoramiento en toda su estructura financiera, motivo por el cual representa una vía de 
canalización para fomentar una estructura de financiamiento admisible para desarrollar la actividad económica de dichas empresas bajo los lineamientos estratégicos financieros de liquidez, endeudamiento y rentabilidad, se espera obtener una proyección que permita estructurar el pasivo a largo plazo en las grandes empresas de la ciudad de Ambato..

\section{Referencias bibliográficas.}

Agronegocios. (7 de diciembre de 2010). Bioalimentar diversifica su producción.

Recuperado el 15 de agosto de 2017 , de http://agronegociosecuador.ning.com/notes/Bioalimentar_diversifica_su_produc ci\%C3\%B3n

Azahar, S., \& Castillo, J. (2006). Investigacion Sobre La Demanda De Credito Bancario Por Parte De Las Pequeñas Y Medianas

Empresas En El Salvador. Trabajo De Graduación, Universidad Centroamericana “Jose Simeon Cañas", El Salvador. Recuperado el 3 de julio de 2017, de http://www.uca.edu.sv/deptos/economia/media/archivo/bf3a02_tesisdemandad ecreditobancarioapymes.pdf

Bautista, M. (2011). La Liquidez Empresarial Y Su Incidencia En La Capacidad De Preducción De La Insdustraia Avícola

Incubandina S.A. En El Periodo 2010. Tesis de ingeniería, Ambato. Obtenido de http://repositorio.uta.edu.ec/bitstream/123456789/1698/1/TA0042.pdf

Bioalimentar. (26 de agosto de 2013). Recuperado el 15 de agosto de 2017, de http://www.bioalimentar.com/index.php/nosotros/mision-y-vision

Bosch, A. (2016). El Concepto De Interés. Recuperado el 3 de julio de 2017, de http://www.antonibosch.com/system/downloads/278/original/EC-

NAVE_Capitulo1.pdf?1297773984

Incubandina S.A. (2018). Incubandina S.A. Recuperado el 2018, de http://incubandina.ec/

Moreta, M. (2017). Expertos en aves de postura. Revista Líderes. Obtenido de http://www.revistalideres.ec/lideres/expertos-aves-postura-empresas- produccion.html

Prieto, C. (2010). Análisis Financiero. Bogotá: Fundación para la Educación San Mateo.

Robles, C. (2012). Fundamentos De Administracion Financiera . Mexico: Red Tercer Milenio S.C.

Rubio, P. (2008). Introducción a la Gestión Empresarial. Madrid: INSTITUTO EUROPEO DE GESTION EMPRESARIAL. 
Solostocks. (2002). Guía para la venta de stocks. Recuperado el 3 de julio de 2017, de Capítulo 3: Impacto financiero en su empresa: http://www.mailxmail.com/curso- guía-ventastocks/impacto-financiero-empresa

SRI. (2017). ¿Quiénes son las personas naturales? Recuperado el 3 de julio de 2017, de http://www.sri.gob.ec/de/31

UNAM. (2016). Las inversiones y los inversionistas. Recuperado el 3 de julio de 2017, de Análisis de riesgo y portafolios de inversión: http://herzog.economia.unam.mx/profesores/blopez/Riesgo-Pres3.pdf

Valladolid Emprende. (2012). El diagnóstico financiero de la empresa: Manuales prácticos de gestión. Recuperado el 4 de Julio de 2017, de http://www.valladolidemprende.es/extras/El_Diagnostico_Financiero_D

E_UNA_EMPRESA.pdf

Veciana, J. (2010). La creación de empresas. Barcelona: La Caixa. Obtenido de http://www.caixabankresearch.com/documents/10180/54279/ee33_esp.pdf

Villalba, M. (2019). Evaluación Del Impacto Financiero En La Gran Empresa De La Ciudad De Ambato En El Año 2016 En Los Créditos Otorgados Por La Corporación Financiera Nacional B.P. Ecuador: Escuela Superior Politécnica de Chimborazo.

Xuletas. (2007). Matemáticas financieras Definiciones. Recuperado el 3 de julio de 2017, de https://www.xuletas.es/ficha/definiciones-74/.

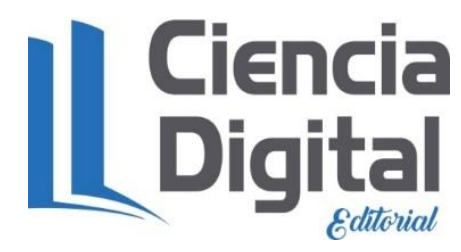




\section{PARA CITAR EL ARTÍCULO INDEXADO.}

Villalba Díaz, M. F. (2020). Evaluación del impacto financiero en la gran empresa de Ambato. ConcienciaDigital, 3(3), 495-509. https://doi.org/10.33262/concienciadigital.v3i3.1345

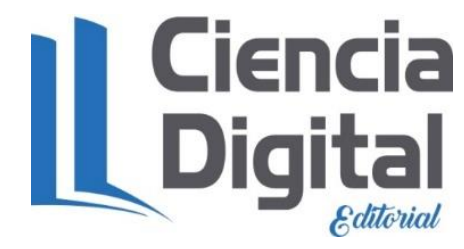

El artículo que se publica es de exclusiva responsabilidad de los autores y no necesariamente reflejan el pensamiento de la Revista Conciencia Digital.

El artículo queda en propiedad de la revista y, por tanto, su publicación parcial y/o total en otro medio tiene que ser autorizado por el director de la Revista Conciencia Digital.
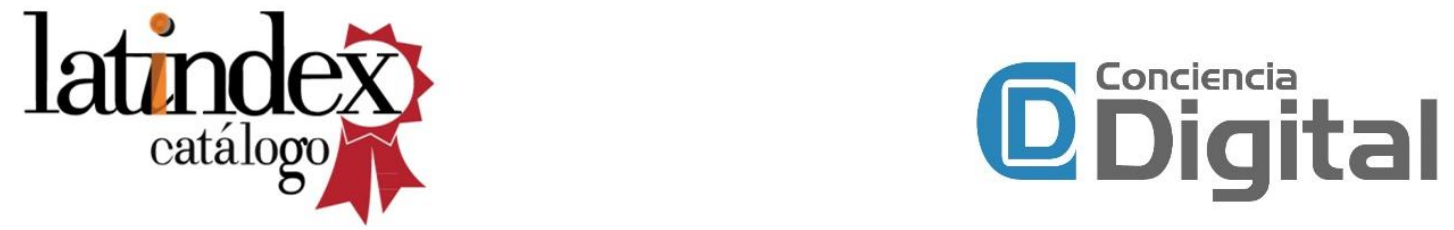